\title{
Modulation of higher-plant NAD(H)-dependent glutamate dehydrogenase activity in transgenic tobacco via alteration of beta subunit levels
}

\author{
Matthew P. Purnell • Damianos S. Skopelitis • \\ Kalliopi A. Roubelakis-Angelakis • José R. Botella
}

Published online: 8 February 2007

(C) Springer-Verlag 2007

\section{Erratum to: Planta 222:167-180}

\section{DOI 10.1007/s00425-005-1510-z}

Unfortunately on page 172 , under the heading $G D H$ isoenzyme profile, $\alpha: \beta$ subunit ratio and sub-cellular localisation of GDH protein in $T_{2}$ transformants, the first pargraph, was published with errors. The correct paragraph is given here:

The GDH isoenzyme profile was examined in A63$\mathrm{H}$ and in each of the three over-expressing lines (Fig. $3 a)$. In roots of the corresponding NS lines, activity staining revealed that the isoenzyme profile was skewed cathodically, suggesting a predominance of $\beta$-subunits. In A63-H roots, there was a slight anodic shift in the isoenzyme profile, suggesting that the amount of $\beta$-subunit may have been reduced. In roots of the over-expressing lines the profile was unexpectedly skewed anodically, with a large increase in the amount of the most anodic isoenzyme. When GDH extracts from leaves of WT tomato and over-expressing line $\mathrm{S} 77-\mathrm{H}$ were co-electrophoresed, the most cathodic tomato isoenzyme (i.e., isoenzyme 1) comigrated with the most anodic isoenzyme of S77-H (Fig. 3b). This indicates that tomato GDH isoenzyme 1 migrates faster than tobacco GDH isoenzyme 1, and that in S77-H tissues the predominant and most anodic isoenzyme is in fact a homohexamer of tomato $\beta$ subunits.

The online version of the original article can be found at http://dx.doi.org/10.1007/s00425-005-1510-z.

\footnotetext{
M. P. Purnell · J. R. Botella $(\varangle)$

Department of Botany,

University of Queensland, Brisbane,

QLD 4072, Australia

e-mail: j.botella@uq.edu.au

D. S. Skopelitis · K. A. Roubelakis-Angelakis

Department of Biology,

University of Crete, P.O. Box 2208,

71409 Heraklion, Greece

Present Address:

M. P. Purnell

BSES Ltd., 50 Meiers Road,

Indooroopilly, QLD 4068, Australia
} 